\title{
Book review: Alles neu, neu, neu! in Afrika: Vier Jahrzehnte Kontinuität und Wandel in der sambischen Provinz
}

\author{
Anna-Sophie Hobi \\ Ethnologisches Seminar, Departement Gesellschaftswissenschaften, Universität Basel, Schweiz \\ Correspondence: Anna-Sophie Hobi (anna-sophie.hobi@unibas.ch)
}

Published: 10 April 2019

Tekülve, M. und Rauch, T.: Alles neu, neu, neu! in Afrika: Vier Jahrzehnte Kontinuität und Wandel in der sambischen Provinz, Verlag Hans Schiler, Berlin/Tübingen, 278 Seiten, ISBN 9783899301205, EUR 19.80 (CHF 27.90 bei Orell Füssli), 2017.

„Alles neu, neu, neu!“ Es habe sich vieles verändert, vieles sei besser geworden, so ein älterer Herr auf die Frage nach den Veränderungen in Kabompo.

Kabompo?

Der kleine Ort im Nordwesten Sambias ist national bekannt für seinen süssen, dunklen Honig. Der gleichnamige Fluss, der sich mäandrierend in die bewaldete Landschaft einfügt, ist zudem der zweittiefste Afrikas, worauf die Leute bei einem Besuch in Kabompo nicht selten mit Stolz hinweisen. Der Distrikt, in welchem heute 110000 Menschen leben (1975 waren es noch 37000 ), ist zwar geografisch abgelegen und dünn besiedelt, verfügt aber über einen lebendigen, betriebsamen und vernetzten Hauptort. Gut $90 \%$ der Bevölkerung sind KleinbäuerInnen. Vermehrt müssen sich SubsistenzbäuerInnen globalen Herausforderungen wie dem Klimawandel oder der Liberalisierung von Agrarmärkten stellen und auch in Sambia mancherorts Bergbaubetrieben oder Grossfarmen weichen. Gleichzeitig sehen Entwicklungsexperten eine nachhaltige und ökologische kleinbäuerliche Landwirtschaft als Rückgrat des globalen Ernährungssystems und als Chance, dem Klimawandel entgegenzuwirken. Doch trägt die Entwicklungszusammenarbeit überhaupt nachhaltig zur Stärkung der KleinbäuerInnen bei oder bleiben von langjährigen Grossprojekten oft nicht mehr als blosse ,Entwicklungs-Ruinen“ übrig?
Genau mit solchen Fragen der ländlichen Entwicklungszusammenarbeit befassen sich Maria Tekülve und Theo Rauch in „Alles neu, neu, neu! in Afrika“. Sie tun dies am Beispiel des kleinbäuerlich geprägten Kabompo im Nordwesten Sambias. Nach 25 Jahren reisen die promovierte Geografin und Entwicklungssoziologin und der habilitierte Wirtschafts- und Sozialgeograf an jenen Ort zurück, wo sie zwischen 1977 und 1993 als Forscherin und als Entwicklungshelfer lebten und arbeiteten. Im Buch reflektieren sie über ihre damaligen Erfahrungen und Erlebnisse und vergleichen sie mit dem heutigen Kabompo.

Bereits im einführenden Kapitel nähern sich die AutorInnen grossen entwicklungspolitischen Debatten, welche auch ausserhalb der Büros von Entwicklungsagenturen oder NGOs polarisierende Diskussionen auslösen. In Anbetracht jahrelanger und wiederkehrender Krisen wird die Hoffnung auf eine positive Wende in Afrika von manchen angezweifelt. Oder sind es doch die KleinbäuerInnen, die zu einem nachhaltigen Wandel beitragen können?

Im in drei Kapitel gegliederten ersten Teil des Buches werfen Tekülve und Rauch dann einen Blick zurück auf die drei Jahrzehnte des partnerorientierten Regionalentwicklungsprogramms der Deutschen Technischen Gesellschaft (GTZ, heute GIZ). Die AutorInnen stellen diesen Beschreibungen in einem umfassenden zweiten Teil die heutige Situation gut 25 Jahre nach Beendigung des Programms gegenüber. Das sechste Kapitel beschreibt anschliessend ein Wiedersehen mit einem ehemaligen sambischen Mitarbeiter, der heute Parlamentsabgeordneter ist. In einer wissenschaftlich und entwicklungspolitisch verankerten Schlussbetrachtung diskutieren Tekülve und Rauch am konkreten Beispiel von Kabompo die am Anfang gestellten grossen Fragen - 
dies, ohne Anspruch auf eine abschliessende Beantwortung dieser zu erheben.

Tekülve und Rauch arbeiteten beide im Rahmen eines Regionalentwicklungsprogramms, welches innerhalb weniger Jahre infolge positiver Wirkung und Beliebtheit zu einem Vorbildprojekt in Sambia wurde. Dieses hatte im Zeitraum von 1977 bis 1993 die Verbesserung der Einkommensmöglichkeiten für Subsistenzbauern und deren Einstieg in die Marktproduktion durch die Organisation der Kleinbäuerinnen sowie die Einführung von Ochsenkarren zum Ziel.

Überdies entstand aus Tekülves Forschung zwischen 1990 und 1993 in Kabompo ihre Dissertation.

Rauch reiste 1977 erstmals nach Sambia und war an der Ausarbeitung des Programms beteiligt. So holprig wie Rauchs erste mehrtägige Reise durch den Kupfergürtel und über Sandpisten in der Nordwestprovinz bis nach Kabompo war, erwies sich auch der Start des Landwirtschaftsprogramms. Dessen Beginn musste aufgrund der skeptischen Haltung und ,antikolonialem Stolz“ der sambischen Verwaltung gegenüber einer aufgedrängten Hilfe, aber auch aufgrund interner regionaler Verteilungskämpfe um zwei Jahre verschoben werden. Packend beschreiben die AutorInnen ihre ersten Eindrücke in Sambia und der Nordwestprovinz und vermitteln dadurch ein Stimmungsbild der lokalen und nationalen Verhältnisse und internationaler Einflüsse, welche auf den ersten Blick besonders in der Hauptstadt Lusaka sichtbar waren. Die ausführliche Beschreibung des Regionalentwicklungsprogramms, gespickt mit Anekdoten und Begegnungen, geben eine leicht nostalgische, aber nicht schönende Darstellung ihres Arbeitsalltags wieder. Gekonnt spannen die AutorInnen immer wieder den Bogen zu den damaligen nationalen und internationalen Veränderungen, welche das abgelegene Kabompo keineswegs unberührt liessen, insbesondere Anfang der 90er-Jahre: Trotz der positiv empfundenen Demokratisierung durch die Einführung des Mehrparteiensystems lösten die HIV/Aids-Epidemie sowie die einschneidenden Strukturanpassungsmassnahmen Frustration und Hoffnungslosigkeit in der Bevölkerung aus. Die Marktliberalisierung brachte grosse Veränderungen für die landwirtschaftliche Produktion mit sich, welche BäuerInnen vorerst dazu zwang, sich neu zu orientieren und zu organisieren. Tekülve verliess Kabompo im Jahr 1993 zwar betrübt, aber doch mit einem kleinen Funken Hoffnung.

Das fünfte und umfassendste Kapitel bildet den Kern des Buches und widmet sich dem Vergleich des damaligen und heutigen Kabompo. Auf einer dreiwöchigen Reise entstanden unzählige Gespräche, Eindrücke und Erlebnisse, welche Tekülve und Rauch im Buch ihren früheren Erfahrungen gegenüberstellen. Damit gelingt den AutorInnen eine spannende Auseinandersetzung, welche sie anhand entwicklungs- und landwirtschaftsrelevanten Themen gliedern und die auch im Kontext ihrer früheren Einsätze relevant waren: Transport, Verbindungen, Elektrizität, Landwirtschaftssystem und Marktproduktion, Bienenhaltung, Holzwirtschaft, Hunger und Ernährung, HIV/AIDS und Gesund- heit sowie auch Geschlechterbeziehungen und Generationenkonflikte. Mit besonderem Stolz erfüllt Tekülve und Rauch die kontinuierliche Verwendung von Ochsengespannen als Transportmittel und als Hilfe bei der Feldbearbeitung.

Den Vergleich schliessen die AutorInnen positiv ab und erkennen klare Verbesserungen in gewissen Bereichen der Lebensverhältnisse in Kabompo. Dazu zählen der Rückgang der Zahl der HIV/Aids-Erkrankungen, eine verbesserte Infrastruktur oder die neuen Technologien der Kommunikation sowie eine leichte Verbesserung der Grundbedürfnisbefriedigung und Ernährungssicherung. Nicht alles ist neu, vieles bleibt laut Tekülve und Rauch gleich: Nach 25 Jahren scheint das Gesamtbild der Dörfer und Siedlungen kaum verändert; eine Existenzgrundlage ausschliesslich basierend auf der lokalen Subsistenzwirtschaft ist noch keineswegs gesichert und erfordert weiterhin ein komplementäres Zusatzeinkommen durch die Lohnarbeit in den Städten, da Einkommensmöglichkeiten und Absatzmärkte in Kabompo instabil bleiben. Besorgniserregend ist zudem die Abholzung der Rosenhölzer für den chinesischen Markt. Der kurze Aufenthalt im Jahr 2015 erlaubt jedoch lediglich einen oberflächlichen und subjektiven Vergleich, was auch die AutorInnen erkennen. Nichtsdestotrotz vermögen sie es, eine interessante und packende gegenüberstellende Analyse zu verfassen.

Durch den Vergleich von damals und heute verfolgen Tekülve und Rauch die Frage nach Kontinuität und Wandel in Kabompo und greifen zugleich grosse entwicklungspolitische Debatten auf. Sie knüpfen an aktuelle Fragen der Entwicklungsforschung an: Ernährungssicherung und insbesondere Landwirtschaft machen zumindest in der Schweiz den grössten Teil der wissenschaftlichen Forschung über die öffentliche Entwicklungshilfe aus und bilden zudem einen Schwerpunkt der schweizerischen sowie deutschen Entwicklungspolitik (BMZ, 2013; Hofmeier, 2016). Im letzten Kapitel werfen die AutorInnen einen kritischen Blick auf die Projekte, an welchen sie mitgewirkt haben. Dies machen sie, ohne dabei die Schwierigkeiten, Enttäuschungen, Misserfolge und Rückschläge zu verheimlichen. Ihre Perspektive beruht auf ihrer gut 40-jährigen Erfahrung in der praktischen Entwicklungszusammenarbeit, welche durch ihre theoretische und wissenschaftliche Auseinandersetzung mit dieser geschärft wird.

Im Bewusstsein der generellen Kritik an Grossprojekten in der Entwicklungszusammenarbeit beurteilen die AutorInnen 'ihr' Programm insgesamt als positiv ohne erkennbare negative Einflüsse und als Auslöser einer gewissen lokalen Dynamik. Dass zentrale Aspekte des Programms noch heute anzutreffen sind, lässt die AutorInnen zum Schluss kommen, dass Entwicklungsprogramme eine unverzichtbare Ergänzung zu einer fairen globalen Wirtschaft und einer verbesserten Regierungsführung sind und dabei besonders die soziale Inklusion berücksichtigen.

In ihrem Buch zeigen Tekülve und Rauch zwar die Debatten rund um die Entwicklungsarbeit auf, stellen diese, genauso wenig wie den Entwicklungsbegriff, aber nicht grund- 
sätzlich in Frage. Dass die dargelegten Debatten typischerweise jenen auf der Nordhalbkugel geführten entsprechen und im Süden teils davon divergierende stattfinden, kommt auch im Gespräch mit dem Parlamentsabgeordneten und früheren Mitarbeiter aus Kabompo zum Vorschein. Dieser versteht Entwicklung in Kabompo nämlich im Sinne von Infrastruktur, Technologie und Wirtschaftsaufschwung. Die sambischen Verständnisse von Entwicklung, so macht es den Anschein, werden von den AutorInnen im Gespräch kritisch aufgefasst. Obwohl die EntwicklungsexpertInnen generell sich an den sambischen Partnern zu orientieren bereit sind, inkludierten sie die lokalen Wünsche im Rahmen ihrer Arbeit im Programm mehr im Sinne eines praktischen Kompromisses als einer grundlegenden Diskussion des Entwicklungsverständnisses. Diese Distanz zwischen SambierInnen und den EntwicklungsexpertInnen aus dem Ausland zeigt sich auch auf persönlicher Ebene: Obwohl man im Rahmen des Programms die Arbeitstage zusammen verbrachte, verfolgte man in der Freizeit meist die eigenen Interessen. Beziehungen waren oft geprägt von ungleichen Einkommensverhältnissen und von einer Lohnabhängigkeit, welche eine Freundschaft mit angestellten SambierInnen gar verunmöglichen sollte. Trotz langen Aufenthalten und der spürbaren Nähe und tiefen Verbundenheit mit Sambia ist dies doch eine ernüchternde Erkenntnis. Die von den AutorInnen beschriebene persönliche und insbesondere entwicklungspolitische Distanz regt dazu an, Partnerschaft und Zusammenarbeit in Entwicklungsprojekten grundlegend zu diskutieren und umzudenken. Denn es stellt sich die Frage, inwiefern die lokalen Bedürfnisse, Vorstellungen und Wünsche in der europäischen Entwicklungspolitik überhaupt Platz finden können.

Tekülves und Rauchs drei Jahrzehnte umspannende Mikrostudie ermöglicht eine längerfristige Betrachtung eines (landwirtschaftlichen) Entwicklungsprojektes und bietet sich durch die authentische und erzählende Darstellung insbesondere LeserInnen an, welche sich der Thematik nähern, aber nicht auf rein statistik- oder theorielastige Werke zurückgreifen möchten. Denn wer die neusten Zahlen und aktuellsten Daten sucht, wird bei der Lektüre weniger zufriedengestellt; das Ergebnis ist vielmehr eine nicht rein wissenschaftliche und doch selbstkritische und spannende Auseinandersetzung mit der praktischen Entwicklungszusammenarbeit. Jemandem, der sich auf das abgelegene Kabompo einlassen und den grünen Nordwesten Sambias - auch nur gedanklich bereisen möchte, verschafft das Buch einen eindrücklichen und treffenden Einstieg. Das Buch richtet sich damit an ein breites Publikum von Sambia- oder Afrika-Interessierten sowie EntwicklungstheoretikerInnen wie auch -praktikerInnen, an „Afro-PessimistInnen“ wie auch „Afro-OptimistInnen“. Durch die narrative Weise gewinnt das Buch eine Vielseitigkeit, woraus eine Art unterhaltsame entwicklungswissenschaftliche Reiseerzählung entsteht. Nicht immer ist klar, welcher der AutorInnen - oder beide - die gerade beschriebene Situation erlebte. Vielmehr stelle man sich ein Gespräch $\mathrm{zu}$ dritt mit den beiden AutorInnen vor - idealerweise bei
Sonnenuntergang auf einer Terrasse hoch über dem mäandrierenden Kabompo-Fluss - in welchem sie die Erzählungen mit aufregenden Geschichten schmücken und sich gegenseitig ergänzen oder auf sympathische Weise gar für den anderen sprechen.

\section{Literatur}

BMZ [Bundesministerium für wirtschaftliche Zusammenarbeit und Entwicklung]: Ernährungssicherung - Strategische Orientierung für die Deutsche Entwicklungspolitik, BMZ-Strategiepapier, Bonn, 11, 15 Seiten, 2013.

Hofmeier, P.: Wichtige Schweizer Akteure in der Entwicklungsforschung, Horizonte, Das Schweizer Forschungsmagazin, 111, S. 20, 2016. 